\title{
La teneur en lysine totale et disponible du lait, des fromages et des produits de boulangerie ayant subi un traitement industriel ${ }^{(*)}$
}

\author{
par \\ A. DE VUYST, W. VERVACK, H. CHARLIER \\ et V. JADIN \\ Centre de Recherches Zootechniques de l'Université de Louvain \\ Lovenjoel (Belgique)
}

\section{INTRODUCTION}

Des expériences antérieures [1] ont montré que le dosage de la lysine totale nous renseigne mal sur la valeur des aliments, si ces aliments ont subi un traitement thermique au cours de leur préparation.

Ce dosage ordinaire, selon la méthode de Spakmann, Stein et Moore [2] est purement quantitatif. Appliqué aux produits alimentaires avant et après traitement industriel, il permet seulement d'apprécier la destruction de lysine. Or, les traitements industriels peuvent non seulement détruire une partie de la lysine, mais également en rendre une autre partie inassimilable par suite du blocage du groupement $\mathrm{E}-\mathrm{NH}_{2}$ de la lysine (réaction de Maillard).

La méthode au fluorodinitrobenzène de Carpenter permet de tenir compte à la fois, de la destruction et du blocage de la lysine. (Nous avons modifié la méthode de Carpenter en substituant au dosage colorimétrique de la dinitrophényllysine, une séparation de ce corps sur colonne d'échangeurs d'ions suivie d'un dosage photométrique après réaction avec la ninhydrine).

Des essais de croissance sur rats et poulets [1] ont montré que la valeur alimentaire d'une ration chauffée n'est pas affectée si l'addition de lysine de synthèse compense exactement la perte de lysine disponible due au chauffage. Si cette addition de lysine de synthèse ne fait que compenser la destruction de lysine, elle est insuffisante pour restituer à la ration chauffée, sa valeur alimentaire primitive.

* Travail subsidié par l'I.B.A.N. (Institut Belge de l'Alimentation et de la Nutrition). 
TABLEAU 1. - Analyses de laits du commerce

\begin{tabular}{|c|c|c|c|c|c|c|c|c|c|}
\hline \multicolumn{2}{|c|}{ Echantillons de lait } & \multirow{2}{*}{\multicolumn{2}{|c|}{$\begin{array}{l}\text { Albumine brute } \\
\text { totale (p. 100) }\end{array}$}} & \multirow{2}{*}{\multicolumn{2}{|c|}{$\begin{array}{l}\text { Lysine totale } \\
\text { (p. } 100-\mathrm{ABT} \text { ) }\end{array}$}} & \multicolumn{4}{|c|}{ Lysine disponible } \\
\hline Nature & Nbre & & & & & (p. 10 & $\mathrm{ABT})$ & (p. $100 \mathrm{l}$ & tot.) \\
\hline Frais & 14 & 3,19 & 0,04 & 9,44 & 0,08 & 9,19 & $\pm 0,12$ & 97,25 & 0,8 \\
\hline Bactofugé & 3 & 3,07 & 0,04 & 9,43 & 0,25 & 8,97 & 0,19 & 95,10 & 1,4 \\
\hline Pasteurisé & 6 & 3,21 & 0,01 & 9,16 & 0,27 & 8,83 & 0,32 & 96,36 & 3,6 \\
\hline Stérilisé & 21 & 3,32 & 0,01 & 8,77 & 0,12 & 8,22 & 0,10 & 94,05 & 1,1 \\
\hline Evaporé & 11 & 7,13 & 0,15 & 8,33 & 0,14 & 5,26 & 0,21 & 63,13 & $2,-$ \\
\hline Condensé sucré & 6 & & & 7,68 & 0,49 & 5,37 & 0,37 & 70,52 & $2,-$ \\
\hline Poudre spray & 13 & 30,29 & 1,60 & 8,96 & 0,11 & 8,29 & 0,18 & 92,60 & $2,-$ \\
\hline Spray enfants & 7 & 17,19 & 0,97 & 8,72 & 0,10 & 7,61 & 0,19 & 87,29 & 2,3 \\
\hline
\end{tabular}


Ces constatations nous ont amené à déterminer dans quelle mesure les traitements industriels subis par le lait, les fromages et les produits de boulangerie, affectent les taux de lysine totale et de lysine disponible de ces produits, en comparaison avec les produits naturels.

\section{1) Le lait}

A) Le tableau 1 rassemble les résultats d'analyses pour les différents types de laits étudiés. Tous ces échantillons proviennent de différentes marques du commerce.

$1^{\circ}$ Albumine : Les variations des teneurs en albumine sont dues aux plus ou moins fortes évaporations d'eau qu'entraînent les différents traitements.

Le lait en poudre Spray pour enfants est du lait en poudre Spray enrichi en lactose.

2० Lysine : Les pertes du lait, tant en lysine disponible qu'en lysine totale, sont proportionnelles à l'importance du traitement thermique subi.

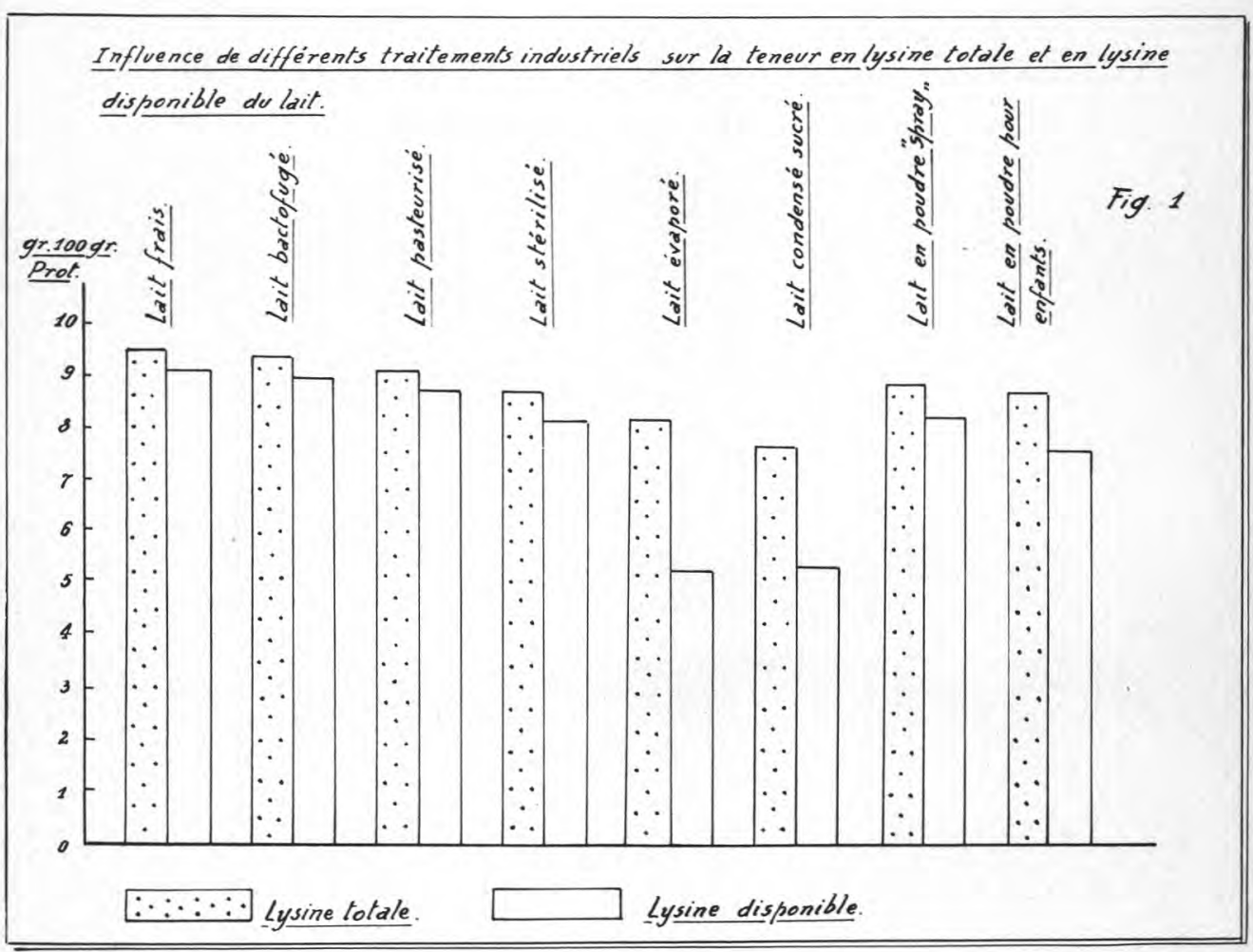


L'analyse statistique des résultats révèle que :

- le lait frais et le lait bactofugé ont des teneurs égales en lysine totale et lysine disponible,

- le lait pasteurisé, qui a subi un traitement thermique léger, ne montre pas de différence significative par rapport au lait frais,

- le lait stérilisé, qui a subi un traitement thermique plus poussé montre une diminution de la teneur en lysine totale et en lysine disponible,

- le traitement industriel encore plus poussé que subissent les laits évaporés et condensés sucrés, provoque une destruction de 12 et 19 p. 100 de la lysine, et un blocage de 29 et 22 p. 100 , soit une détérioration totale de 41 p. 100 par rapport à la lysine du lait frais,

- le lait en poudre spray (évaporation rapide sous vide) n'a pas subi de perte aussi élevée et se rapproche du lait stérilisé au point de vue teneur en lysine totale et disponible,

- le lait en poudre diététique renferme moins de lysine disponible que le lait en poudre spray.

\section{Conclusion}

a) la stérilisation et la fabrication de lait en poudre spray font perdre au lait environ 9 p. 100 de sa lysine disponible,

b) les laits évaporés et condensés sucrés perdent 40 p. 100 de la lysine disponible du lait frais.

(Le dosage de la lysine totale nous donne seulement une perte de 12 p. 100).

B) Le tableau 2 récapitule les pertes de lysine (totale et disponj. ble) encourues par le lait si nous le laissons pendant $1 / 2,1,2$ et $4 \mathrm{~h}$ en présence ou non de différents sucres.

TABLEAU 2. - Influence de la durée d'ébullition et de l'addition de sucre

\begin{tabular}{|c|c|c|c|c|c|c|}
\hline Durée d'ébullition & \multicolumn{2}{|c|}{ (En heures) } & $1 / 2$ & 1 & 2 & 4 \\
\hline Lait frais & $\begin{array}{l}\text { destruction } \\
\text { blocage } \\
\text { détérioration }\end{array}$ & $\begin{array}{l}\text { p. } 100 \\
\text { p. } 100 \\
\text { p. } 100\end{array}$ & $\begin{array}{r}2 \\
8 \\
10\end{array}$ & $\begin{array}{r}7 \\
11 \\
18\end{array}$ & $\begin{array}{l}16 \\
10 \\
26\end{array}$ & $\begin{array}{l}19 \\
14 \\
33\end{array}$ \\
\hline Lait +5 p. 100 glucose & $\begin{array}{l}\text { destruction } \\
\text { blocage } \\
\text { détérioration }\end{array}$ & $\begin{array}{l}\text { p. } 100 \\
\text { p. } 100 \\
\text { p. } 100\end{array}$ & $\begin{array}{r}11 \\
6 \\
17\end{array}$ & $\begin{array}{r}20 \\
9 \\
29\end{array}$ & $\begin{array}{r}33 \\
7 \\
40\end{array}$ & $\begin{array}{l}43 \\
19 \\
62\end{array}$ \\
\hline Lait +5 p. 100 lactose & $\begin{array}{l}\text { destruction } \\
\text { blocage } \\
\text { détérioration }\end{array}$ & $\begin{array}{l}\text { p. } 100 \\
\text { p. } 100 \\
\text { p. } 100\end{array}$ & $\begin{array}{r}7 \\
6 \\
13\end{array}$ & $\begin{array}{l}11 \\
12 \\
23\end{array}$ & $\begin{array}{l}24 \\
15 \\
39\end{array}$ & $\begin{array}{l}27 \\
23 \\
50\end{array}$ \\
\hline Lait +5 p. 100 saccharose & $\begin{array}{l}\text { destruction } \\
\text { blocage } \\
\text { détérioration }\end{array}$ & $\begin{array}{l}\text { p. } 100 \\
\text { p. } 100 \\
\text { p. } 100\end{array}$ & $\begin{array}{l}4 \\
2 \\
6\end{array}$ & $\begin{array}{c}11 \\
3 \\
14\end{array}$ & $\begin{array}{r}14 \\
6 \\
20\end{array}$ & $\begin{array}{l}16 \\
15 \\
31\end{array}$ \\
\hline
\end{tabular}


Nous appelons :

- destruction: la perte de lysine totale,

- détérioration : la perte de lysine disponible,

- blocage : la partie de la détérioration qui n'est pas due à une destruction pure et simple de lysine, mais à un blocage de son groupement $\mathrm{E}-\mathrm{NH}_{2}$.

$1^{\circ}$ La détérioration de la lysine est croissante si la durée d'ébullition est prolongée. Cette détérioration croissante de la lysine est due, à la fois, à une destruction et à un blocage.

$2^{\circ}$ L'influence de l'addition de sucres dépend, quant à elle, de la nature des sucres ajoutés.

Si ces sucres sont réducteurs (glucose et lactose), leur action sur la détérioration de la lysine est très nette :

- le glucose influençant davantage la destruction,

- le lactose influençant davantage le blocage.

Si les sucres ajoutés ne sont pas réducteurs (saccharose), ils ne modifient pas la perte de lysine au cours de l'ébullition.

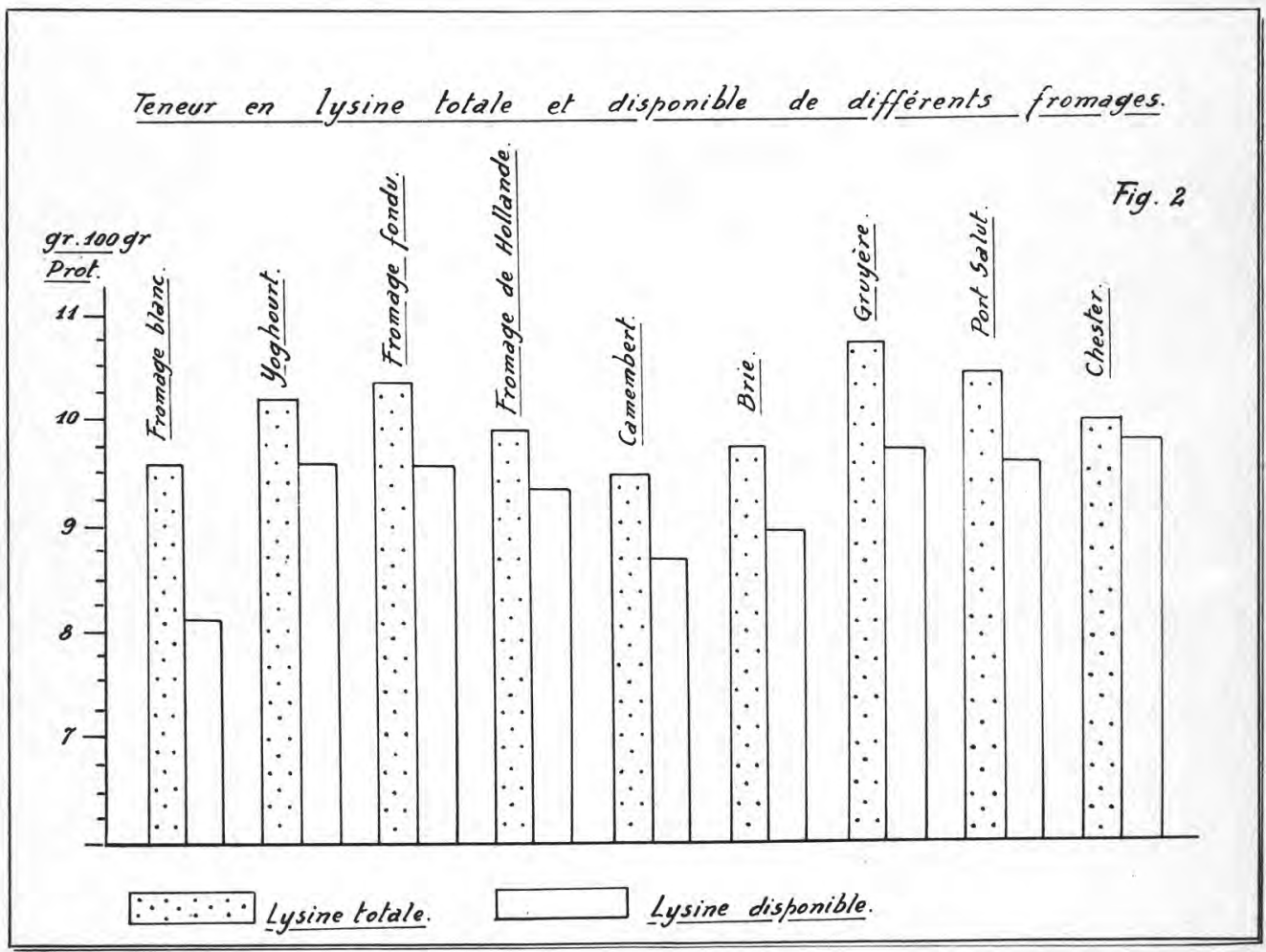


TABLEAU 3.- Analyses de fromages

\begin{tabular}{|c|c|c|c|c|c|c|c|c|}
\hline \multicolumn{2}{|c|}{ Echantillons } & \multirow{2}{*}{\multicolumn{2}{|c|}{$\begin{array}{l}\text { Albumine } \\
\text { brute totale } \\
\text { (p. 100) }\end{array}$}} & \multirow{2}{*}{\multicolumn{2}{|c|}{$\begin{array}{c}\text { Lysine } \\
\text { totale } \\
\text { (p. } 100 \mathrm{ABT})\end{array}$}} & \multicolumn{3}{|c|}{ Lysine disponible } \\
\hline Nature & Nombre & & & & & (p. 10 & ABT) & $\begin{array}{c}(\text { p. } 100) \\
\text { lysine totale }\end{array}$ \\
\hline Yoghourt & 18 & $4,45 \pm$ & 0,15 & 9,25 & 0,13 & 8,61 & $=0,26$ & 93,1 \\
\hline Fromage blanc & 19 & 9,10 & 0,36 & 8,68 & 0,13 & 7,13 & 0,33 & 82,1 \\
\hline Fromage fondu & 16 & 14,86 & 0,68 & 9,37 & 0,14 & 8,60 & 0,24 & 91,8 \\
\hline Camembert & 7 & 21,46 & 0,72 & 8,56 & 0,76 & 7,73 & 0,19 & 90,3 \\
\hline Brie & 6 & 20,68 & 0,57 & 8,83 & 0,21 & 8,01 & 0,41 & 90,7 \\
\hline Chester & 2 & 20,47 & & 9,06 & & 8,82 & & 97,4 \\
\hline Port Salut & 4 & 25,29 & 1,16 & 9,52 & 0,51 & 8,65 & 0,74 & 90,9 \\
\hline Tilsit & 2 & $25,-$ & & 8,46 & & 8,62 & & $100,-$ \\
\hline Hollande & 6 & 26,33 & 0,76 & 8,92 & 0,31 & 8,39 & 0,42 & 94,1 \\
\hline Gruyère & 6 & 30,10 & 0,54 & 9,81 & 0,42 & 8,56 & 0,68 & 87,3 \\
\hline
\end{tabular}




\section{2) Les fromages}

Au tableau 3, figurent les teneurs en lysine totale et disponible du yoghourt et des principaux types de fromages rencontrés sur le marché.

$1^{\circ}$ Albumine : Le taux d'albumine varie à peu près proportionnellement au taux de matière sèche des différents fromages.

20 Lysine: La teneur en lysine totale des fromages à pâte molle (Camembert et Brie) est inférieure à celle des autres fromages ; la maturation longue de ces fromages favorise l'activité des désaminases d'origine microbienne, qui vont dégrader quelque peu la plupart des acides aminés.

L'étude de la disponibilité de la lysine dans ces fromages nous a permis, dans deux cas seulement, d'observer un blocage plus prononcé de cet acide aminé :

- dans le cas du fromage blanc, particulièrement riche en lactose,

- dans le cas du Gruyère qui subit une pasteurisation du caillé.

3o Conclusion : La composition en acides aminés de la protéine du lait n'est guère modifiée par la transformation du lait en yoghourt ou en fromages.

Les fromages à pâte molle (Camembert et Brie) subissent une légère perte d'acides aminés et notamment de lysine.

La disponibilité de la lysine est supérieure à 90 p. 100, sauf pour le fromage blanc et le Gruyère.

\section{3) La farine de blé et les produits de boulangerie}

Le tableau 4 rassemble les résultats d'analyses effectuées sur ces produits pour lesquels la détermination du taux de lysine disponible revêt une importance particulière, puisque la lysine y est largement limitante.

$1^{\circ}$ Albumine : Il convient de signaler que certains de ces produits contiennent, en plus ou moins grande quantité, d'autres sources de protéines que la farine, et notamment du lait, des œufs et de la levure.

2० Lysine : La teneur en lysine du produit de base est faible, mais cette lysine est presque entièrement disponible. Cette disponibilité :

- reste entière dans la mie de pain,

- diminue dans la mie des pistolets : l'épaisseur du produit mis au four est moindre que dans le cas du pain,

- diminue encore dans la mie des sandwichs : les sandwichs contiennent davantage de sucre que le pain et les pistolets,

- est nettement plus faible dans la croûte que dans la mie,

- est de l'ordre de 70 p. 100 dans les biscottes et la chapelure qui sont chauffées en vue de leur séchage, 
TABLEAU 4. - Analyses de farine et de produits dérivés

\begin{tabular}{|c|c|c|c|c|c|}
\hline \multicolumn{2}{|c|}{ Echantillons } & \multirow{2}{*}{$\begin{array}{l}\text { Albumine } \\
\text { brute totale } \\
\quad(p .100)\end{array}$} & \multirow{2}{*}{$\begin{array}{c}\text { Lysine } \\
\text { totale } \\
\text { (p. } 100 \mathrm{ABT} \text { ) }\end{array}$} & \multicolumn{2}{|c|}{ Lysine disponible } \\
\hline Nature & Nbre & & & (p. 100 ABT) & (p.100 lys. tot.) \\
\hline Farine blanche & 10 & 11,27 & 2,05 & $2,-$ & 97 \\
\hline Pain mie & 10 & 10,72 & 2,32 & 2,33 & 98 \\
\hline Pain croûte & 10 & 11,80 & 1,61 & 1,23 & 75 \\
\hline Pistolets mie & 10 & 11,38 & 2,48 & 2,20 & 90 \\
\hline Pistolets croûte & 10 & 12,61 & 1,91 & 1,41 & 76 \\
\hline Sandwich mie & 5 & 10,56 & 2,32 & 1,69 & 72 \\
\hline Sandwich croûte & 5 & 11,66 & 1,99 & 1,10 & 56 \\
\hline Biscotte & 6 & 11,87 & 1,25 & 0,95 & 70 \\
\hline Chapelure & 3 & 12,02 & 1,81 & 1,19 & 67 \\
\hline Cake & 4 & 7,65 & 2,95 & 2,42 & 80 \\
\hline Petit beurre & 6 & 8,28 & 1,70 & 1,30 & 71 \\
\hline Pain d'épices & 6 & 3,07 & 1,23 & 0,28 & 25 \\
\hline Spéculoos & 7 & 5,46 & 0,86 & 0,29 & 46 \\
\hline
\end{tabular}


Influence de différentes préparations industrielles sur les teneurs en lysine totale et en lysine disponible de la farine et de produits a base de farine.

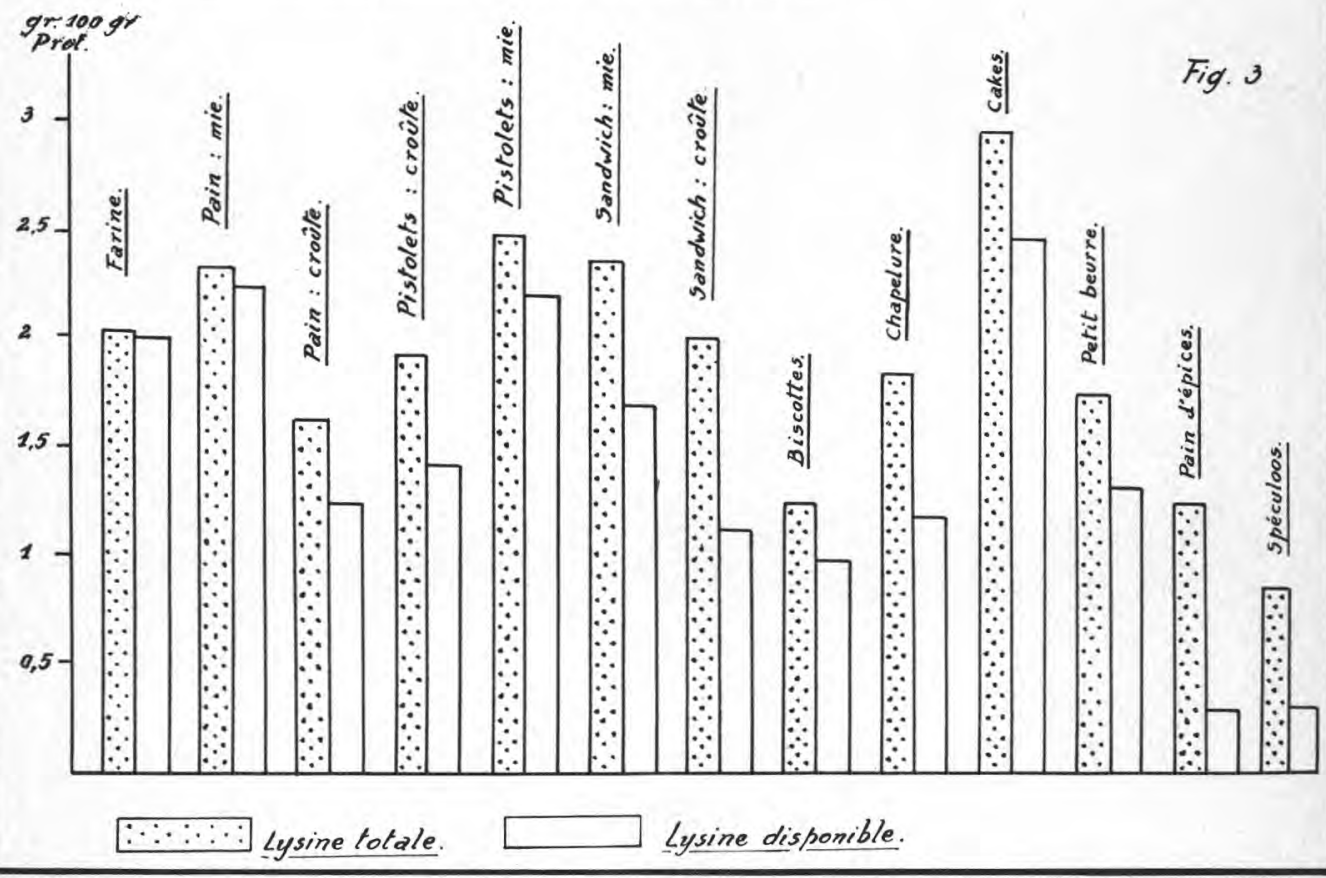

- reste de l'ordre de 70 à 80 p. 100 dans les cakes et les petits beurres malgré la présence de sucres ; ceci serait dû au rôle protecteur de la graisse qui est plus abondante dans ces friandises,

- est extrêmement réduite dans le cas des spéculoos et des pains d'épices : la présence très importante de sucres réducteurs et le traitement thermique favorisent la réaction de Maillard.

3o Conclusion : La lysine est totalement disponible dans le produit de base, mais cette disponibilité est affectée dans la mesure où le traitement thermique et la présence de sucres réducteurs sont importants.

\section{Rés u m é}

Nous avons étudié l'influence du traitement industriel des produits laitiers (lait et fromages) et des produits de boulangerie sur leur teneur en lysine totale et disponible.

L'analyse montre que l'influence néfaste du traitement industriel est d'autant plus accusée que la température est plus élevée au cours 
du traitement, que la durée de chauffage est plus longue, que le taux de sucres réducteurs est plus important.

\section{S u m mary}

Influence of industrial treatments of dairy-milk and cheese and bakers' products on their content in total and available lysin has been studied. Analysis shows that the baleful influence of industrial treatment is greather when the temperature is higher during treatment, the heating time longer and the rate of reducing sugars more important.

Reçu pour publication en février 1972.

\section{Références bibliographiques}

De Vuyst (A.), Vervack (W.) Charlier (H.) et Jadin (V.). - Influence du traitement thermique sur la valeur biologique de la protéine des aliments. Bull. de l'Académie de Médecine de Belgique, 1971.

Spackman (D. H.), Moore (S.) and SteIn (W. H.). - Analytical Chemistry, 30, 1158, 1958. 